Monatsschrift f. Geburtshülfe u. Gynäkologie 1924;66:317-318

\title{
Der Ausschuß für Frauenwohl in New York
}

Unter Mitwirkung der New Yorker geburtshilflichen Gesellschaft und der dortigen Akademie für Medizin ist im März 1923 aus führenden Kreisen ein Komitee zusammengetreten für ,, Maternal Health” - also für das Wohl der Frauen. Als eine der ersten Aufgaben hat sich dieses Komitee eine klinische Studie über Schwangerungsverhütung und Kontrazeption gestellt. Monatssohrift f. Geburtshülfe u. Gynäkologie. Bd. LXVI. Heft 4’5.21 318 Referate aus geburtshülfiich-gynäkologisclien Gesellschaften.

Es sollen die Probleme der Fertilität und Sterilität untersucht werden; davon also zunächst das einer therapeutischen Verhütung der Konzeption. Es gilt entsprechende ärztliche Berichte zu sammeln und kritisch zu sichten, ohne Beziehung auf Behandlung oder Beratung aus der Literatur oder dureh Umfrage bei erfahrenen Fachleu†en. Dabei sol] das Verfahren der gewollten Schwängerungsverhütung weitgehend zum Studium herbei-gezogen werden. In Betracht kommen nur die staatlich erlaubten Vor-beugungsmaßna·hmen zur Verhütung oder Behandlung. Urn das Studium zu fördern, sollen, wahrscheinlich mit finanzieller Unterstützung, dem Komitee entsprechende Patienten zugeführt werden. Sie müssen von an-erkannter, ärztlicher Seite bezügliche Berichte über Diagnose und Indikation mitbringen. Dann werden sie einer anerkannten Klinik überwiesen, deren Stab für die therapeutischen Maßnahmen verantwortlich ist. Die darüber eingesandten Berichte werden gesammelt. Die Patienten werden in ihrem Verhalten weiter beobachtet. Das Komitee behält sich die entsprechende Veröffentlichung vor. Eine ärztliche Gruppe bearbeitet die medizinischen Fragen, eine juridische die legalen, eine die finanziellen. Staatlich angestellte Pflegerinnen sollen die Patienten betreuen und beobachten. Andere Gruppen sollen in alien Richtungen die sozialen Seiten des Problems unterstützen. Der ausführende Ausschuß, der sich nach Bedarf durch Kooptation ergänzen kann, trägt die voile Verantwortung.

Die Aufnahme der bisherigen Vorschläge war nicht einheitlich; es bedarf noch weiterer Aufklärung im Sinne des Programms.

Wie wir hören, soil in den Niederlanden eine ähnliche Bewegung be-stehen. Das Komitee hat zum eingehenden Studium derselben einen Experten dorthin gesandt.

Wir können dem Komitee nur unsere voile Sympathie ausdrücken und dem großzügig geplanten Unternehmen einen durchgreifenderen Erfolg wünschen, als wie bisher gelegentliche Aussprachen in ärztlichen Gesell schaften befürchten lassen. A. Martin.

II.

Referate

aus

geburtshülflich-gynäkologischen Gesellschaften.

Amerikanische Association der Geburtshelfer, Gynäkologen und Abdominalchirurgeri.

Philadelphia. 19.-20. IX. (Am. J. obst. gynec. VII. 2. S. 206.) Keefe: Über Hospitaler. Chandler: Die Durchtrennung der Ileozökalfalte als eine Maßnahme bei der 
Appendizitisoperation. - Farr: Lokalanästhesie bei der Behandlung septischer Erkrankungen im Abdomen. - Wellon: Der Zeitpunkt für die Operation bei ektopischer Schwangerschaft. Morris: Vorsicht beim Operieren. - Rosegg: Der Nutzen der Strahlen bei

Menstruationsstörungen. - Reder: Verletzung des Stumpfes des supravaginal amputierten Uterus. - Lynch: Diverticula und Diverticulitis. - Bainbridge: Uterus duplex mit multipler Schwanger- 